\title{
A comparative study of automatic techniques for ocular artifact reduction in spontaneous EEG signals based on clinical target variables: A simulation case
}

\author{
Sergio Romero $^{\mathrm{a}, *}$, Miguel A. Mañanas ${ }^{\mathrm{a}}$, Manel J. Barbanoj ${ }^{\mathrm{b}}$ \\ ${ }^{a}$ Department of Automatic Control (ESAII), Biomedical Engineering Research Center, Technical University of Catalonia (UPC), 08028 Barcelona, Spain \\ ${ }^{\mathrm{b}}$ Drug Research Center (CIM), Research Institute of Sant Pau Hospital, Department of Pharmacology and Therapeutics, Autonomous University of \\ Barcelona (UAB), 08193 Barcelona, Spain
}

Received 14 March 2007; accepted 3 December 2007

\begin{abstract}
Eye movement artifacts represent a critical issue for quantitative electroencephalography (EEG) analysis and a number of mathematical approaches have been proposed to reduce their contribution in EEG recordings. The aim of this paper was to objectively and quantitatively evaluate the performance of ocular filtering methods with respect to spectral target variables widely used in clinical and functional EEG studies. In particular the following methods were applied: regression analysis and some blind source separation (BSS) techniques based on second-order statistics (PCA, AMUSE and SOBI) and on higher-order statistics (JADE, INFOMAX and FASTICA). Considering blind source decomposition methods, a completely automatic procedure of BSS based on logical rules related to spectral and topographical information was proposed in order to identify the components related to ocular interference. The automatic procedure was applied in different montages of simulated EEG and electrooculography (EOG) recordings: a full montage with 19 EEG and 2 EOG channels, a reduced one with only 6 EEG leads and a third one where EOG channels were not available. Time and frequency results in all of them indicated that AMUSE and SOBI algorithms preserved and recovered more brain activity than the other methods mainly at anterior regions. In the case of full montage: (i) errors were lower than $5 \%$ for all spectral variables at anterior sites; and (ii) the highest improvement in the signal-to-artifact (SAR) ratio was obtained up to $40 \mathrm{~dB}$ at these anterior sites. Finally, we concluded that second-order BSS-based algorithms (AMUSE and SOBI) provided an effective technique for eye movement removal even when EOG recordings were not available or when data length was short.
\end{abstract}

(C) 2007 Elsevier Ltd. All rights reserved.

Keywords: Electroencephalography (EEG); Electrooculography (EOG); Ocular filtering; Regression analysis; Blind source separation (BSS); Principal component analysis (PCA); Independent component analysis (ICA)

\section{Introduction}

Quantitative analysis and interpretation of human electroencephalographic (EEG) signals are useful methods to study functional states of the brain, to evaluate drug effects and to diagnose psychiatric and neurological disorders. The most widely used techniques to quantify changes in EEG signals are based on spectral analysis and topographic mapping. In humans,

\footnotetext{
* Corresponding author at: Dept. d'Enginyeria de Sistemes, Automàtica i Informàtica Industrial, Centre de Recerca en Enginyeria Biomèdica, Universitat Politècnica de Catalunya, C/Pau Gargallo 5, 08028 Barcelona, Spain. Tel.: +3493401 1689; fax: +34934017045.

E-mail address: sergio.romero-lafuente@upc.edu (S. Romero).
}

EEG power is traditionally divided into spectral broad frequency bands related to EEG generators and rhythms: delta, theta, alpha and beta.

Electrical scalp recordings do not contain only cerebral activity (EEG) but also non-cortical electrical activities, such as artifacts of physiological and non-physiological origin. Physiologic artifacts are generated from the subject and the ocular activity is the most important. Eye movements and blinking are a major source of EEG contamination because they occur very frequently and the electrooculographic (EOG) signal amplitude can be several times larger than brain scalp potentials. Their vertical ocular projection propagates quite symmetrically in a anterior-posterior direction and it contaminates the recorded EEG signals. Lateral eye movements mainly affect 
lateral frontal electrodes [1]. The potentials generated by ocular activity interfere with the electric field of neural origin mainly, in the anterior scalp regions. To a certain extent, neural activity especially from the anterior scalp area is also recorded by electrodes placed near the eyes. EEG and EOG recordings are therefore a mixture of brain and ocular electrical activity. This is known as bidirectional contamination.

A critical point in EEG signal processing is the need for careful treatment and reduction of these artifacts which contaminate the EEG signals and can lead to wrong results and conclusions.

The aim of the study was to objectively and quantitatively evaluate the performance of filtering methods based on linear regression and component analysis with respect to spectral target variables and topographic maps, both widely used in clinical and functional EEG studies. For this purpose, two different EEG channel configurations were used and even the absence of EOG recordings was also considered.

The most common reduction method is based on linear regression in the time or frequency domain. This method estimates and removes the influence of EOG on the signals recorded by scalp electrodes from the EEG recordings. However, this technique has an inherent drawback that is the aforementioned bidirectional contamination. In other words, regression procedures consider that the EEG recording is not correlated with the EOG recording, and this assumption is generally erroneous. Therefore, relevant cerebral information contained in the EOG recordings can also be cancelled in the EEG recordings corrected by means of regression techniques [2]. Some studies suggest that most of the high frequency range in the EOG is of neural origin. Thus, in order to reduce the cancellation of interesting cerebral information, low-pass filtering of the EOG signals has been proposed before the application of a time domain regression approach [3]. Furthermore, a recent study suggests that ocular artifact effects in EEG signals are not only present in delta and theta ranges, but also in alpha and beta bands, so the above-mentioned filtering does not seem to be an adequate solution [4].

Other approaches for ocular artifact reduction are based on a linear decomposition of the EEG and EOG recordings into source components, the so-called blind source separation (BSS) techniques. These component-based methods permit the identification of artifactual sources, and then the reconstruction of EEG recordings without these sources. The first proposed component-based procedure was the principal component analysis (PCA) [5]. By means of a rotation, PCA transforms a multi-variable data set into components which are spatially orthogonal. The main drawback with PCA is the assumption of orthogonality between neural activity and ocular artifact and this is not generally true. Lagerlund et al. reported that PCA could not completely separate eye artifacts from cerebral activity, especially if they have similar amplitudes [6].

In order to overcome the limitations and constraints presented by regression and PCA procedures newer approaches use blind source extraction based on independent component analysis (ICA). The assumption taken into account in these approaches is that sources must be statistically independent, not just uncorrelated and this is mostly true with regard to the brain and ocular components. ICA is currently considered an important technique for removing artifacts from EEG signals [2,7,8]. However, most of the studies detect visually artifactual components following a subjective criterion. In order to overcome this subjectivity, few studies have been carried out using a completely automatic artifact correction process based on statistical properties like kurtosis, entropy or correlation with EOG channels [9-11]. Many articles use these filtering approaches in real EEG data. However, the quantitative evaluation of the goodness of each correction method is very difficult because it is necessary to know which are the true brain and ocular components. To date, few sets of algorithms based on simulated data have been evaluated in order to solve this problem [10,12]. Furthermore, opposite results were obtained: in the former study, the best performance was obtained with an ICA algorithm [10], while in the latter, ocular artifact correction methods based on regression and PCA performed better than ICA-based correction procedures [12]. Besides, some of the ICA algorithms that were evaluated and inadvisable in [10] require much longer data segments than the epoch duration considered in that study in order to work properly [13]. In addition, the approach on which the results of [10] were based, an eye tracker procedure, is quite different from the clinical routine, where spontaneous EEG signals are commonly acquired with closed eyes and with normal (not fast forced) eye movements. Moreover, another recent study defended regression-based methods against BSS when the number of EEG leads was small [14]. Concerning this point, a reduced number of electrode configuration or even without EOG leads (where linear regression approach and automatic procedures based on correlation with EOG channels are not applicable) have not been yet analyzed. Finally, the effects of filtering results on the spectral bands widely used as target variables and on the distribution of the correction effectiveness through the scalp topography have not been analyzed either.

This study takes into account all the questions mentioned above and proposes a completely automatic BSS procedure in simulated EEG and EOG recordings with different durations, where the cerebral and ocular components are previously known, in order to simulate real clinical situations.

\section{Methodology}

\subsection{Subjects and instrumentation}

Forty healthy volunteers, 20 males and 20 females, aged between 20 and 32 years (mean age 23.75 years) were selected for the study from a larger database corresponding to clinical EEG studies with closed eyes. Twenty volunteers with normal eye movements were selected following inspection of EOG signals. The remaining 20 volunteers were carefully chosen based on the criterion of no apparent eye movements. Spontaneous EEG and EOG signals, sampled at $100 \mathrm{~Hz}$, were recorded during a 3-min vigilance-controlled condition with eyes closed. During the acquisition, the experimenter kept the volunteers alert by means of acoustic stimulation as soon as drowsiness patterns appeared in the EEG signals. 
The vertical EOG (VEOG) signal was recorded from midforehead $(2.5 \mathrm{~cm}$ above the pupil) to the average of one electrode below the left eye and one electrode below the right eye ( $2.5 \mathrm{~cm}$ below the pupil). The horizontal EOG (HEOG) signal was obtained from the outer canthi. EEG signals were recorded from 19 electrodes placed on the scalp according to the international 10-20 system on the following locations: Fp1, Fp2, F7, F3, Fz, F4, F8, T3, C3, Cz, C4, T4, T5, P3, Pz, P4, T6, $\mathrm{O} 1$ and $\mathrm{O} 2$, referenced to averaged mastoids [15]. The amplifiers used were neuroscan synamps. The signals were recorded using a band pass filter between 0.3 and $50 \mathrm{~Hz}$.

\subsection{Simulated data}

As the propagation of the signals (EEG and EOG) is very fast, a simultaneous linear mixture between source EEG $\left(\mathrm{EEG}_{\mathrm{s}}\right)$ and $\mathrm{EOG}\left(\mathrm{EOG}_{\mathrm{s}}\right)$ signals is proposed in order to simulate a real case [12]. Twenty 3-min epochs were simulated according to an instantaneous mixing model between the $\mathrm{EEG}_{\mathrm{s}}$ and $\mathrm{EOG}_{\mathrm{S}}$ coming signals expressed by

$$
\left(\begin{array}{c}
\mathrm{EOG}_{\mathrm{m}} \\
\mathrm{EEG}_{\mathrm{m}}
\end{array}\right)=A\left(\begin{array}{c}
\mathrm{EOG}_{\mathrm{s}} \\
\mathrm{EEG}_{\mathrm{s}}
\end{array}\right),
$$

where the subindexes $\mathrm{m}$ and $\mathrm{s}$ denote mixed and source signals. Simulated data was created by mixing $\mathrm{EEG}_{\mathrm{s}}$ and $\mathrm{EOG}_{\mathrm{s}}$ coming from different subjects in order to guarantee independence between them.

These propagation factors were used as the weights of the mixing matrix $A$ which contaminates the $E_{E G}$ signals. Next, VEOG and HEOG signals from this available data were lowpass filtered with a cut-off frequency of $7.5 \mathrm{~Hz}$ in order to guarantee that the majority of potentials were of ocular origin [3]. These filtered signals were considered VEOG and HEOG sources.

EEG sources were considered following the criterion of low ocular activity. One 3-min epoch was selected from each one of the remaining 20 subjects in the database. The criterion to select a certain segment was that no samples of the VEOG or MEOG exceeded $40 \mu \mathrm{V}$ during the $3 \mathrm{~min}$. EEG sources were obtained by high-pass filtering all the 19 EEG derivations with a cut-off frequency of $0.5 \mathrm{~Hz}$ in order to guarantee that very low frequency components were removed.

Ocular contamination was simulated by means of the addition of VEOG and HEOG sources (weighted by their corresponding propagation factors) to the EEG sources which composed the $\mathrm{EEG}_{\mathrm{m}}$ signals. Neural contamination by cerebral activity was then simulated by the partial contribution of the average fronto-polar ( $\mathrm{Fp} 1$ and $\mathrm{Fp} 2)$ and lateral frontal (F7 and F8) activities which composed $\mathrm{VEOG}_{\mathrm{m}}$ and $\mathrm{HEOG}_{\mathrm{m}}$ signals, respectively. The partial contribution of the $\mathrm{EEG}_{\mathrm{s}}$ into the $\mathrm{EOG}_{\mathrm{m}}$ was assumed to be the same propagation factor as that from the $\mathrm{VEOG}_{\mathrm{s}}$ or $\mathrm{HEOG}_{\mathrm{s}}$ into the $\mathrm{EEG}_{\mathrm{m}}$ signal. Eq. (2) describes the global mixing process followed in order to obtain $\mathrm{EOG}_{\mathrm{m}}$ and $\mathrm{EEG}_{\mathrm{m}}$ signals.

$$
\left[\begin{array}{c}
\mathrm{VEOG}_{\mathrm{m}} \\
\mathrm{HEOG}_{\mathrm{m}} \\
\mathrm{Fp} 1_{\mathrm{m}} \\
\mathrm{Fp} 2_{\mathrm{m}} \\
\mathrm{F} 7_{\mathrm{m}} \\
\mathrm{F} 8_{\mathrm{m}} \\
\vdots \\
\alpha_{\mathrm{Fp} 1}
\end{array}\right]=\left[\begin{array}{ccc}
1 & \beta_{\mathrm{Fp} 1} & 1 \\
\alpha_{\mathrm{Fp} 2} & \beta_{\mathrm{Fp} 2} & 0 \\
\alpha_{\mathrm{F} 7} & \beta_{\mathrm{F} 7} & 0 \\
\alpha_{\mathrm{F} 8} & \beta_{\mathrm{F} 8} & 0 \\
\vdots & \vdots & \vdots \\
\alpha_{O 2} & \beta_{O 2} & 0
\end{array}\right.
$$

$\left.\begin{array}{cccccc}\alpha_{\mathrm{Fp} 2} / 2 & 0 & 0 & 0 & \cdots & 0 \\ 0 & \beta_{\mathrm{F} 7} / 2 & \beta_{\mathrm{F} 8} / 2 & 0 & \cdots & 0 \\ 0 & 0 & 0 & 0 & \cdots & 0 \\ 1 & 0 & 0 & 0 & \cdots & 0 \\ 0 & 1 & 0 & 0 & \cdots & 0 \\ 0 & 0 & 1 & 0 & \cdots & 0 \\ \vdots & \vdots & & \ddots & & \vdots \\ 0 & 0 & 0 & \cdots & & 1\end{array}\right]\left[\begin{array}{c}\mathrm{VEOG}_{\mathrm{s}} \\ \mathrm{HEOG}_{\mathrm{s}} \\ \mathrm{Fp} 1_{\mathrm{s}} \\ \mathrm{Fp} 2_{\mathrm{s}} \\ \mathrm{F} 7_{\mathrm{s}} \\ \mathrm{F} 8_{\mathrm{s}} \\ \vdots \\ \mathrm{O} 2_{\mathrm{s}}\end{array}\right]$

Regarding the ocular activity, one 3-min epoch with vertical and horizontal eye movements was selected from each one of the first 20 subjects in the database. Then, individual vertical $(\alpha)$ and horizontal $(\beta)$ propagation factors, representing the proportion of the amount of EOG that is present in a particular EEG channel, were calculated for each EEG channel by means of linear regression using all this 3-min epoch. That is, measured EEG signals were considered dependent variables and measured VEOG and HEOG signals were used as independent variables in the linear regression. This regression was carried out for each of the 20 subjects in order to obtain 20 sets of propagation factors that reflected different real situations of ocular interferences. For the procedure, only samples with high VEOG or HEOG amplitude were used to calculate the sum of squares of regression analysis [16]. where $\alpha_{\mathrm{EEG}}$ and $\beta_{\mathrm{EEG}}$ represent the vertical and horizontal ocular propagation factors, respectively, which are calculated using the EEG channel denoted by the subindex. Mixing procedure by means of Eq. (2) was carried out 20 times in order to simulate 20 subjects or sets of mixed signals. In each case, $\mathrm{EOG}_{\mathrm{S}}$ signals with their corresponding propagation factors and $\mathrm{EEG}_{\mathrm{s}}$ were used. A schematic diagram of the simulation process is shown in Fig. 1.

\subsection{Regression-based approaches to ocular artifacts reduction}

The multiple linear regression method in the time domain which was applied in this study was based on the methodology described in Semlitsch et al. [17]. Individual regression factors ( $\gamma$ for $\mathrm{VEOG}_{\mathrm{m}}$ and $\delta$ for $\mathrm{HEOG}_{\mathrm{m}}$ ) were calculated for each 


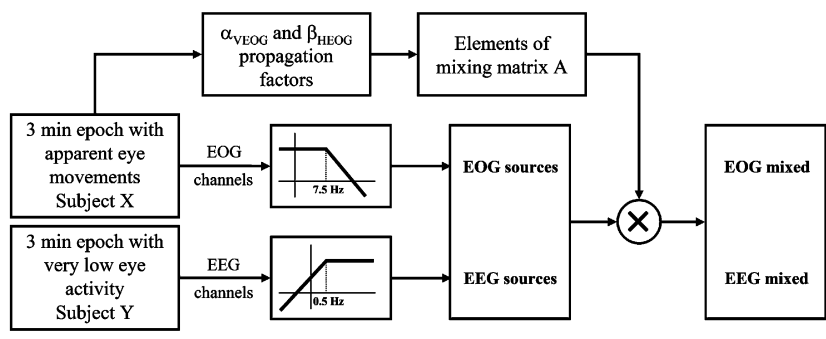

Fig. 1. Simulation procedure representing a linear mixing of real EEG and EOG signals corresponding to different subjects.

$\mathrm{EEG}_{\mathrm{m}}$ channel

$\gamma=\frac{\sum_{i=1}^{N} \operatorname{VEOG}_{\mathrm{m}}(i) \cdot \operatorname{EEG}_{\mathrm{m}}(i)}{\sum_{i=1}^{N} \operatorname{VEOG}_{\mathrm{m}}^{2}(i)} ;$

$\delta=\frac{\sum_{i=1}^{M} \operatorname{HEOG}_{\mathrm{m}}(i) \cdot \mathrm{EEG}_{\mathrm{m}}(i)}{\sum_{i=1}^{M} \mathrm{HEOG}_{\mathrm{m}}^{2}(i)}$,

where $N$ and $M$ represent the number of samples used for the calculation of $\gamma$ and $\delta$, respectively.

In order to improve the estimation of these factors, only samples with high $\mathrm{VEOG}_{\mathrm{m}}$ or $\mathrm{HEOG}_{\mathrm{m}}$ amplitudes were used to calculate the sum of squares for regression analysis [16].

Regression methods assume that the recorded EEG signals $\left(E E G_{m}\right)$ are a linear, time-invariant superposition of different sources. The corrected EEG $\left(E_{E} G_{c}\right)$ signals were obtained by removing the portion of $\mathrm{VEOG}_{\mathrm{m}}$ and $\mathrm{HEOG}_{\mathrm{m}}$ which was present in the $\mathrm{EEG}_{\mathrm{m}}$

$\mathrm{EEG}_{\mathrm{c}}(t)=\mathrm{EEG}_{\mathrm{m}}(t)-\gamma \cdot \operatorname{VEOG}_{\mathrm{m}}(t)-\delta \cdot \mathrm{HEOG}_{\mathrm{m}}(t)$.

It is known that this method also removes part of the cerebral activity which is present in the $\mathrm{EOG}_{\mathrm{m}}$ channels [2]. To reduce this cancellation of cerebral information, some studies suggest a low-pass filtering (cut-off frequency of $7.5 \mathrm{~Hz}$ ) of $\mathrm{EOG}_{\mathrm{m}}$ signals before the application of the regression procedure [3]. This option was also considered to be evaluated in this study with the name of 'filtered regression' method.

\subsection{Spatial component approaches to ocular artifacts reduction}

BSS methods consider the parametric estimation of the following generative model for the data:

$x=A \cdot s$,

where $x$ and $s$ are matrices composed by $n$ and $m$ row vectors, respectively, and $A$ is a $n \times m$ matrix which must be estimated. In other words, there are mixtures $x_{i}(0<i \leqslant n)$ composed by original source signals $s_{j}(0<j \leqslant m)$. Nothing is known about the mixing process $A$ or the sources $s_{j}$ whose estimation is the objective of BSS.

\subsubsection{Uncorrelated sources}

Second-order statistics (SOS) techniques only consider the assumption that source signals are uncorrelated in order to estimate the mixing matrix and the original sources. Three techniques were considered in this study: PCA, algorithm for multiple unknown signals extraction (AMUSE) and second-order blind identification (SOBI). The PCA procedure calculates a rotated orthogonal matrix $A$ in such a way that mixtures in the new coordinates become uncorrelated. In other words, PCA obtains a low-dimensional representation of data which contains most of their variance. AMUSE [18] and SOBI [19] belong to the group of spatio-temporal decorrelation procedures with SOS. These algorithms are sometimes classified as ICA techniques [20]. In fact, uncorrelation is a weak form of statistical independence. As EEG and EOG data have temporal structures, less restrictive conditions than statistical independence are often sufficient to estimate the mixing matrix and sources. Basics of AMUSE and SOBI algorithms consist of simultaneous diagonalization of the corresponding lagged covariance matrix (using one time lag for AMUSE and several lags for SOBI).

\subsubsection{Independent sources}

Higher-order statistics (HOS) are crucial to solve the BSS problem when original sources are assumed to be statistically independent. This independence is a more general concept than decorrelation: two random variables are statistically independent if knowledge of the values of one variable provides no information about the values of the other one. There are several procedures to measure statistical independence, basically based on approaches of non-gaussianity, mutual information and maximum likelihood estimation [20].

In this study, three common ICA methods, with their variations, were evaluated: JADE [21], INFOMAX [22] and FASTICA [23]. The JADE algorithm is based on the joint approximate diagonalization of eigenmatrices defined by fourth-order cumulant tensors. The INFOMAX principle consists of the maximization of the joint entropy of a neural processor output, that is the same as minimizing the mutual information among the output components. Besides, in this work we applied an extended-INFOMAX version [24], which performs the decomposition on linear mixtures with either subor super-gaussian distributions. Finally, FASTICA is a computationally efficient implementation based on a maximization of the non-gaussianity given by the negentropy.

ICA algorithms require a sufficient number of samples to provide reliable results. A minimum number corresponding to a few times the square of the number of channels is usually needed to obtain reliable decompositions [13]. However, stationarity of the signal cannot be assumed when long time segments are used. Thus, additional data points improve the decomposition of HOS algorithms only if relative stationarity of the spatial structure of the EEG and EOG sources can be assumed. In order to evaluate this effect, different duration of epochs of $E G_{m}$ and $E_{m}$ signals were considered when component based methods, SOS and HOS algorithms, were applied: 5, 10, $15,30,60,90,120$ and $180 \mathrm{~s}$.

Decomposition procedures were performed using the functions included in the ICALAB toolbox v2.2 for Matlab [25]. 


\subsubsection{Automatic selection of artifact-related components}

In Eq. (5), rows of the observed matrix $x$ were the simulated $\mathrm{EEG}_{\mathrm{m}}$ and $\mathrm{EOG}_{\mathrm{m}}$ signals recorded at different electrodes. Once the components were estimated by means of any of the algorithms mentioned above $(\hat{s}=W \cdot x)$, the rows of the matrix $\hat{s}$ were the estimated source signals $s_{j}$ (independent components: IC), and the columns of the inverse matrix $W^{-1}$, which was the estimation of $A$, provided the projection strengths of each IC onto the scalp sensors. Therefore, scalp topographies of the IC allowed examination of the biological origin and to decide whether or not an IC was related to an artifact.

Thus, automatic artifact identification was based on frequency and scalp topography aspects of the IC. The criteria to remove an IC related to an eye movement artifact were simultaneously:

(i) Relative power in delta band in excess of a certain high percentage.

(ii) Projection strength (normalized to the maximum) on the EOG electrodes above a certain threshold. This condition was related to the correlation between IC and EOG channel.

(iii) Projection strength on the EEG electrodes following a gradient related to the propagation of the ocular artifact that is empirically known [26]. These values had to decrease in the following order: fronto-polar, frontal, central, parietal and occipital areas in the case of vertical movement; and frontal, central, parietal and occipital areas in the case of horizontal movement.

(iv) Maximum projection strength on the EEG electrodes higher than a threshold, whose value depended on whether the interference corresponded mainly to a vertical or a horizontal eye movement.

After detecting IC related to EOG interference, corrected EEG was reconstructed from the remaining components by zeroing out the corresponding rows of the matrix $\hat{s}$.

\subsection{Validation of correction}

Frequency variables often used in clinical EEG studies were selected in order to evaluate the correction effect of each technique described above. Spectral analysis was performed for all EEG channels. Power spectral density (PSD) functions were calculated by means of periodogram using a Hanning window of 5-s duration [16]. Several variables were calculated from the PSD functions: total power $(0.5-35 \mathrm{~Hz})$, and absolute and relative power of the following bands [27]: delta $(0.5-3.5 \mathrm{~Hz})$, theta $(3.5-7.5 \mathrm{~Hz})$, alpha $(7.5-13 \mathrm{~Hz})$ and beta $(13-35 \mathrm{~Hz})$. Relative variables were calculated with respect to the total power. These band power values were calculated for further comparison before and after EOG correction. Percentage errors in each spectral variable were calculated between the sources (true values) and all the corrected EEG channels. Furthermore, the mean square error (MSE) between sources and corrected EEG signals was calculated for all the methods.

\section{Results}

\subsection{Simulated data}

Results in the time domain of the simulation procedure can be observed in Fig. 2. Fig. 2a shows an example of $5 \mathrm{~s}$ epoch corresponding to EEG sources from one volunteer and EOG sources from another, in order to guarantee that there is no contamination between them. Fig. 2b shows the mixed EEG and EOG data obtained multiplying the sources by the corresponding weight matrix applying Eq. (1). The bidirectional contamination between cerebral and ocular activity can be observed in Fig. 2b, especially in the VEOG and fronto-polar (Fp1 and Fp2) channels.

Elements of the 20 mixing matrices applied to simulate the propagation of the ocular activity over the scalp were obtained from the vertical and horizontal regression factors calculated for each subject who provided the EOG sources. Table 1 shows VEOG and HEOG propagation factors in mean and standard deviations for the 20 subjects related to the sources of ocular activity.

VEOG propagation factors were maximal at the fronto-polar electrodes (Fp1 and Fp2), decreasing from anterior to posterior channels. HEOG interference had opposite signs for left and right hemispheres, obtaining the maximal propagation factors in absolute values at lateral-frontal channels (F7 and F8).

Signal-to-artifact ratio (SAR) was computed for each EEG channel in order to evaluate the extent of ocular contamination over the scalp. Thus, this index was defined as the ratio between the total power of the true cerebral component (EEG source) and the total power of the true EOG component (corresponding to the propagation of both vertical and horizontal ocular activity to that channel). This true EOG component could be rewritten as the MSE between the mixed (contaminated) EEG signal and the EEG source.

$$
\begin{aligned}
& \text { SAR }_{\mathrm{EEG}} \\
& =10 \cdot \log \frac{\frac{\sum_{i=1}^{N}\left(\mathrm{EEG}_{\mathrm{m}}(i)\right)^{2}}{N}}{\frac{\sum_{i=1}^{N}\left(\alpha_{\mathrm{EEG}} \cdot \operatorname{VEOG}_{\mathrm{s}}(i)+\beta_{\mathrm{EEG}} \cdot \mathrm{HEOG}_{\mathrm{s}}(i)\right)^{2}}{N}} \\
& =10 \cdot \log \frac{\operatorname{Energy}\left\{\mathrm{EEG}_{\mathrm{s}}\right\}}{\mathrm{MSE}\left\{\mathrm{EEG}_{\mathrm{m}}-\mathrm{EEG}_{\mathrm{s}}\right\}},
\end{aligned}
$$

where $\alpha_{\mathrm{EEG}}$ and $\beta_{\mathrm{EEG}}$ were the vertical and horizontal propagation factors, respectively, for this EEG channel, and $N$ was the number of samples. Fig. 3 shows a scalp map with the SAR calculated by averaging the values obtained for the 20 simulated mixed data. Lower SARs were obtained in anterior channels and the index increased toward the posterior scalp sites. The almost total symmetry of the map was logical.

\subsection{Effect of artifact filtering on time courses}

Fig. 4 shows three EEG channels (Fp1, F7 and C3) corresponding to the same $5 \mathrm{~s}$ interval shown in Fig. 2, before and 

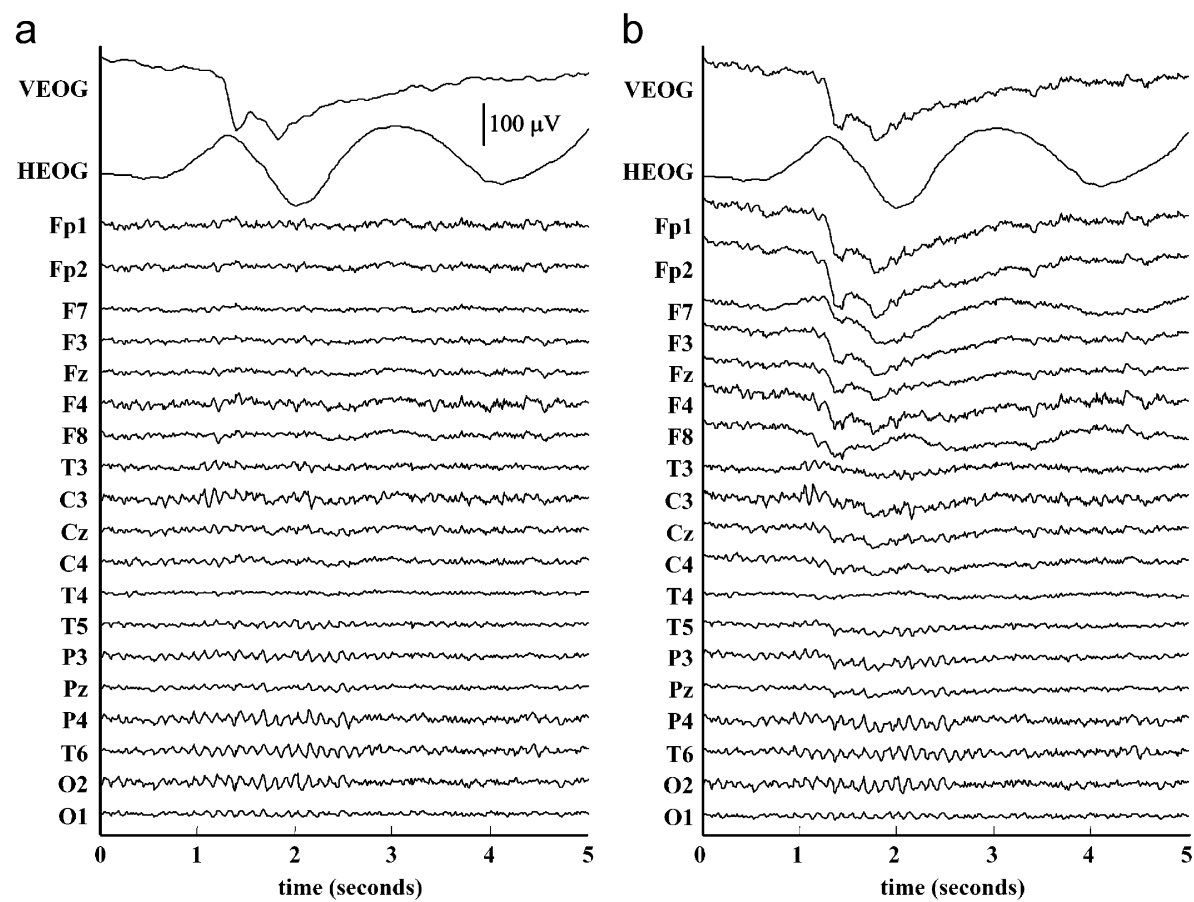

Fig. 2. (a) Five seconds epoch corresponding to EEG and EOG sources from two different volunteers. (b) Five seconds segment corresponding to mixed EOG and EEG signals obtained after applying the linear mixing simulation procedure to the sources shown in (a). Mixed EEG signals contained a prominent slow eye movement.

Table 1

Vertical and horizontal EOG regression factors for the 20 subjects whose $\mathrm{EOG}_{\mathrm{s}}$ were extracted for the mixture process (mean $\pm \mathrm{std}$ )

\begin{tabular}{llr}
\hline Channel & \multicolumn{1}{l}{ VEOG } & \multicolumn{1}{l}{ HEOG } \\
\hline Fp1 & $0.969 \pm 0.075$ & $0.029 \pm 0.046$ \\
Fp2 & $0.983 \pm 0.083$ & $-0.063 \pm 0.041$ \\
F7 & $0.439 \pm 0.142$ & $0.309 \pm 0.055$ \\
F3 & $0.495 \pm 0.101$ & $0.085 \pm 0.027$ \\
Fz & $0.409 \pm 0.095$ & $-0.008 \pm 0.022$ \\
F4 & $0.463 \pm 0.107$ & $-0.103 \pm 0.026$ \\
F8 & $0.405 \pm 0.143$ & $-0.300 \pm 0.036$ \\
T3 & $0.104 \pm 0.046$ & $0.114 \pm 0.026$ \\
C3 & $0.223 \pm 0.069$ & $0.073 \pm 0.025$ \\
Cz & $0.202 \pm 0.069$ & $-0.001 \pm 0.022$ \\
C4 & $0.221 \pm 0.081$ & $-0.073 \pm 0.023$ \\
T4 & $0.114 \pm 0.073$ & $-0.113 \pm 0.031$ \\
T5 & $0.005 \pm 0.108$ & $0.027 \pm 0.026$ \\
P3 & $0.113 \pm 0.056$ & $0.030 \pm 0.024$ \\
Pz & $0.094 \pm 0.052$ & $-0.001 \pm 0.024$ \\
P4 & $0.050 \pm 0.025$ & $-0.033 \pm 0.019$ \\
T6 & $0.053 \pm 0.035$ & $-0.035 \pm 0.017$ \\
O1 & $0.027 \pm 0.029$ & $0.009 \pm 0.023$ \\
O2 & $0.027 \pm 0.029$ & $-0.007 \pm 0.021$ \\
\hline
\end{tabular}

after applying the different ocular removal techniques. Visual effectiveness of the proposed correction procedures on different EEG channels can be observed. Comparing the sources with the corrected EEG signals by means of regression-based techniques, this approach also reduced neural activity recorded in EOG data. This effect was especially located at anterior EEG channels because of the bidirectional contamination between

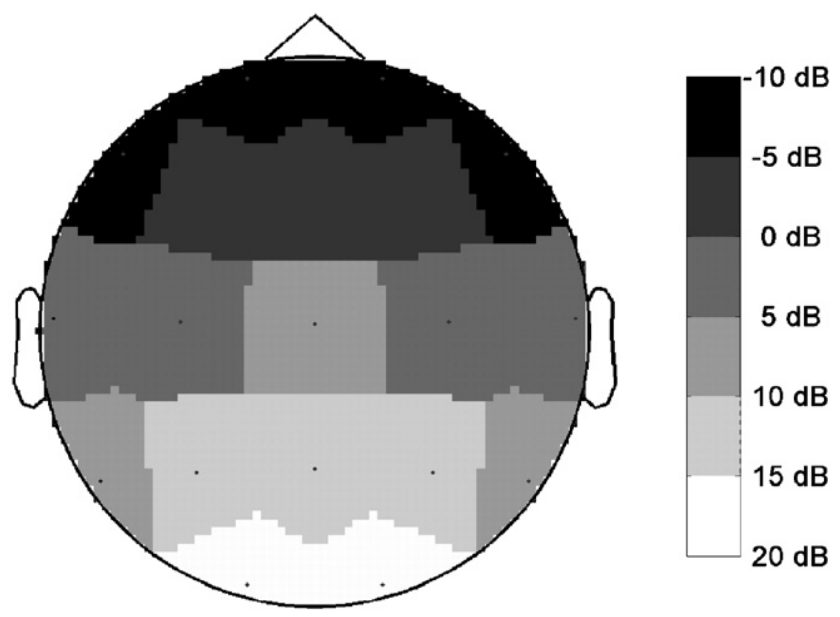

Fig. 3. Scalp topography of the averaged SAR ratio. Dark grey represent negative values of SAR (high ocular contamination) and light grey depict positive SAR values (weak ocular contamination).

ocular and cerebral activity. PCA procedure also removed interesting cerebral activity at anterior channels. In spite of HOSbased techniques eliminated quite accurately ocular artifacts as the rest of the methods, corrected EEG signals showed modified cerebral activity in frontal sited locations with respect to $\mathrm{EEG}_{\mathrm{s}}$ signals. By visual inspection, SOBI algorithm produced more similar corrected EEG signals to the original source than other methods at the fronto-polar (Fp1) and the lateral-frontal (F7) channels. Very similar results were obtained for the AMUSE algorithm, indicating that SOS techniques extracted eye movement artifacts with less loss of cerebral information. 
a

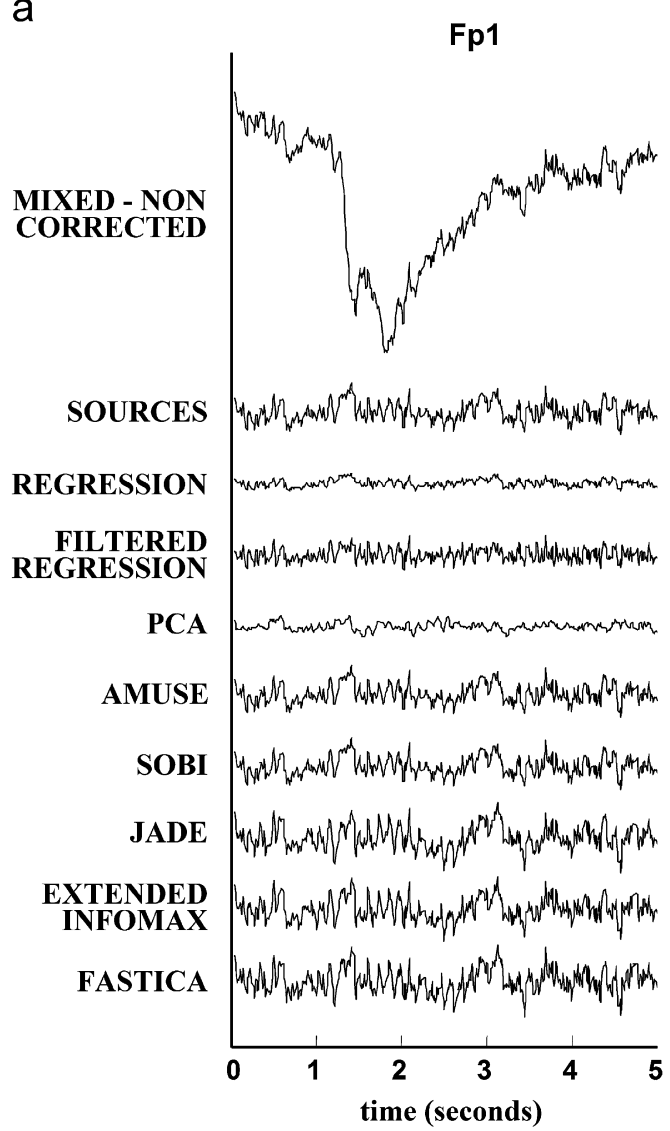

b

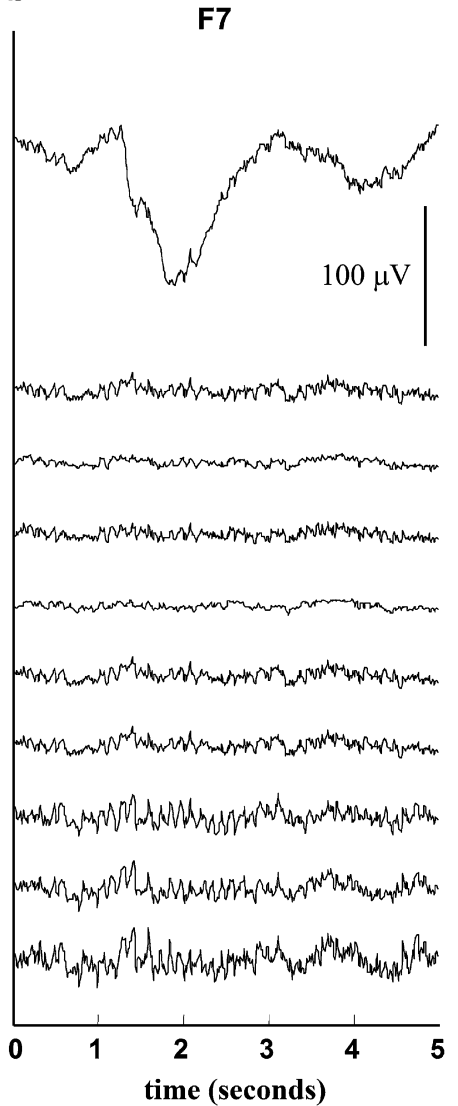

C

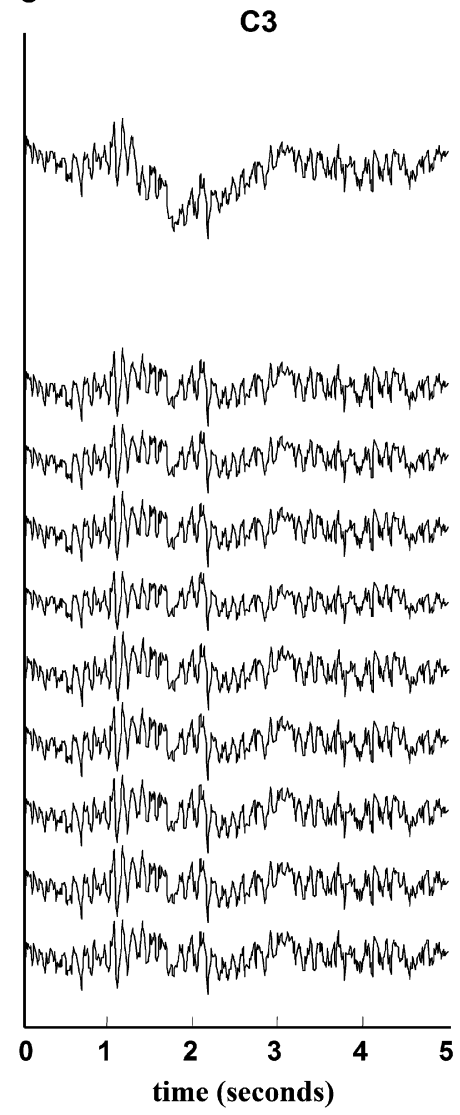

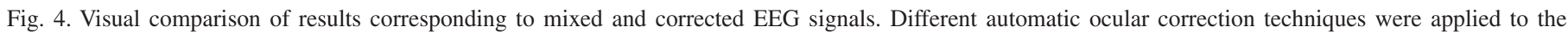

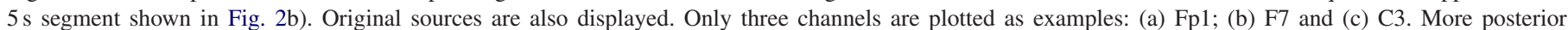
channels showed a better performance for all the correction methods since ocular incidence is lower than in anterior channels.

No apparent visual differences between ocular correction approaches were observed for the central and more posterior channels.

\subsection{Validation of correction}

\subsubsection{Target variables}

BSS was applied using different epoch durations: 5, 10, $15,30,60,90,120$ and $180 \mathrm{~s}$. For the regression procedures, the propagation factors only depended on the subject and the electrode location at the scalp. Therefore, these factors were obtained considering all the available $180 \mathrm{~s}$ in each simulated subject. If only few seconds including ocular artifacts were used for their computation, it was difficult to obtain reliable factors because there were not enough eye movements for the estimation [16]. Percentage errors between the EEG sources and the corrected EEG signals were calculated for each filtering technique and for all spectral variables. These errors, in absolute and relative powers of delta, theta, alpha and beta bands, are shown in Fig. 5. Mean values for all the 19 EEG channels and all the 20 simulated data are presented using different duration of epochs for the blind separation in the component-based procedures. Errors for AMUSE algorithm are not depicted because they were very similar to errors obtained for SOBI algorithm. Besides, similar percentage errors were obtained for AMUSE and SOBI in all different epoch durations. Errors increased slightly with higher durations for PCA. However, errors decreased by increasing the epoch length of the data in HOS-based techniques (JADE, INFOMAX and FASTICA). Percentage errors for AMUSE and SOBI were lower than for the other approaches in all epoch durations.

Table 2 shows the percentage errors averaged across channels obtained with different EOG correction procedures and using the epoch duration which provided the minimum error in each technique: $5 \mathrm{~s}$ for PCA decomposition, $90 \mathrm{~s}$ for AMUSE and SOBI and 180-s epochs for HOS-based algorithms. Percentage errors for non-corrected EEG signals are also included.

The spectral band with the highest error in non-corrected EEG signals was the delta band where there was the main incidence of ocular contamination in the EEG channels. All the correction methods reduced percentage errors obtained for the mixed (no corrected) EEG signals in absolute and relative delta band. Errors for absolute alpha and beta powers obtained by regression analysis were high due to additional removal of neural activity which was common to the EOG and EEG channels. These errors were reduced after applying the filtered version of the regression technique. 

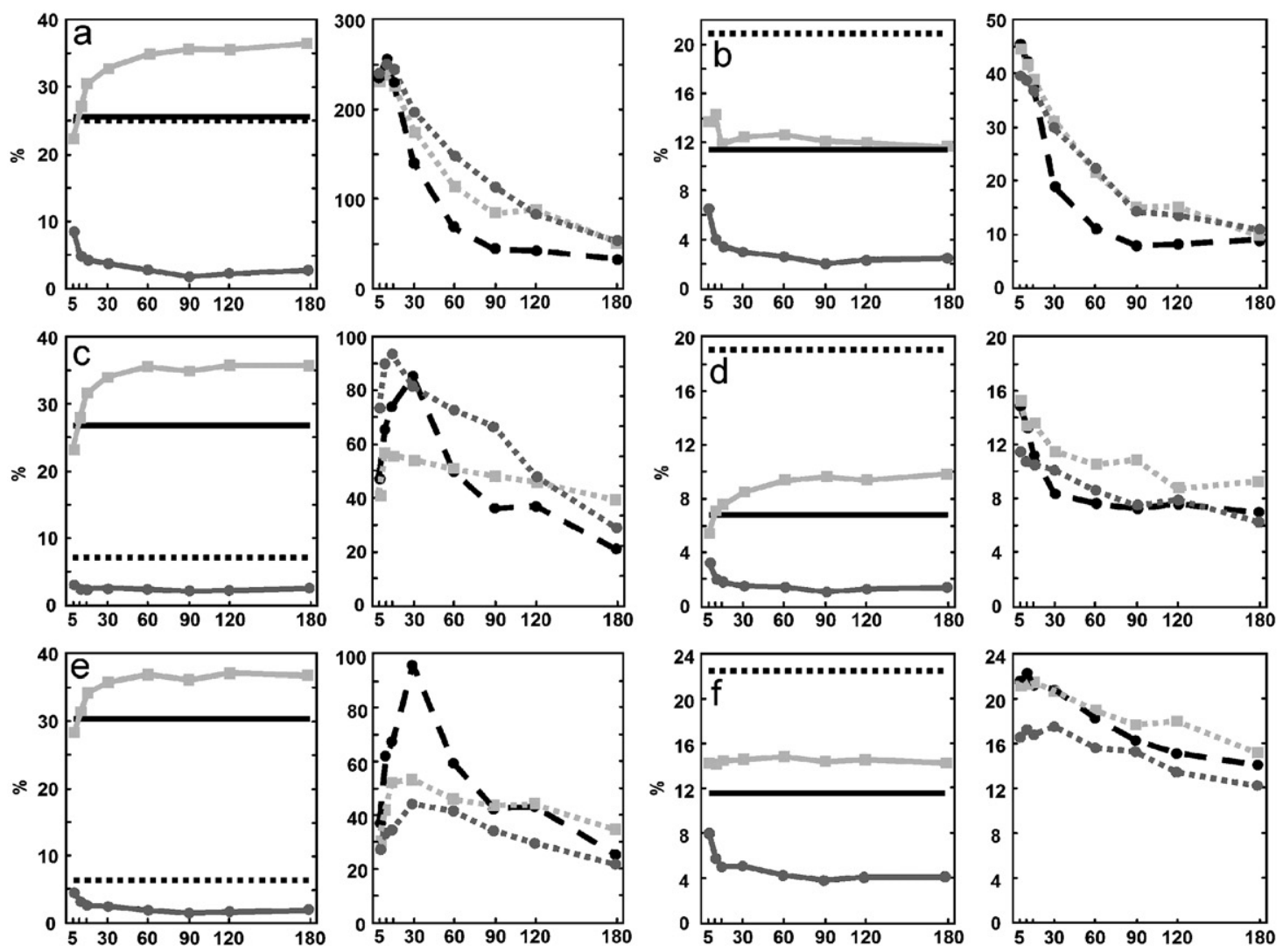

Regression

Filtered Regression
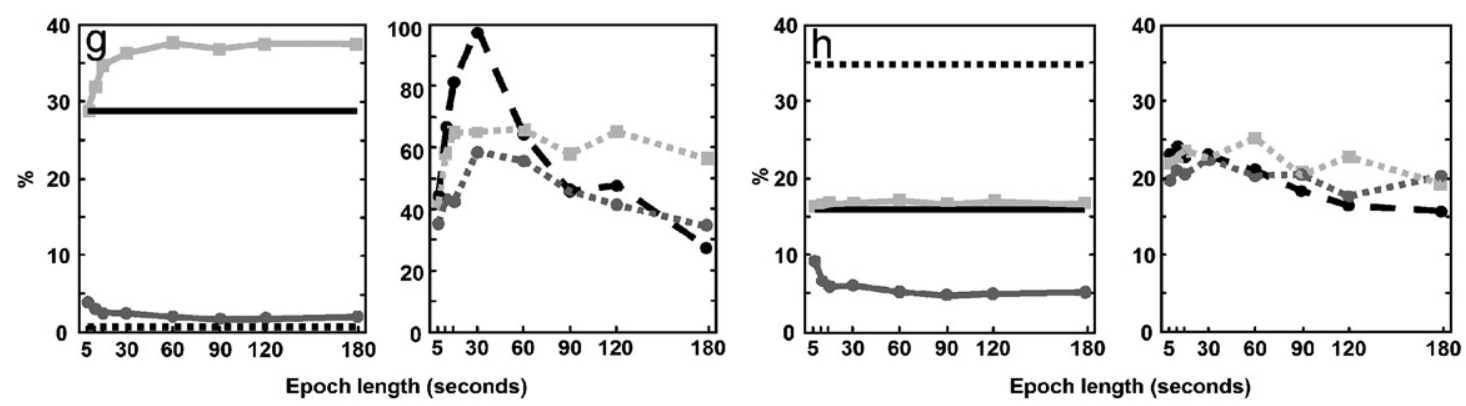

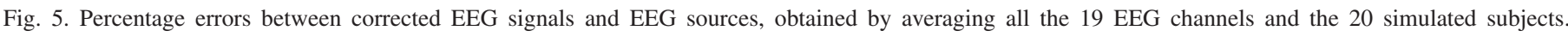

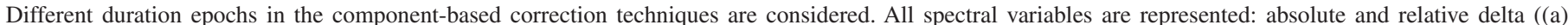

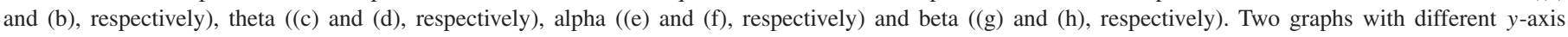
scale are used because of the different range of values between HOS-based methods (right graph) and the others (left graph).

Table 2

Percentage errors $(\%)$ in spectral variables for EOG correction procedures

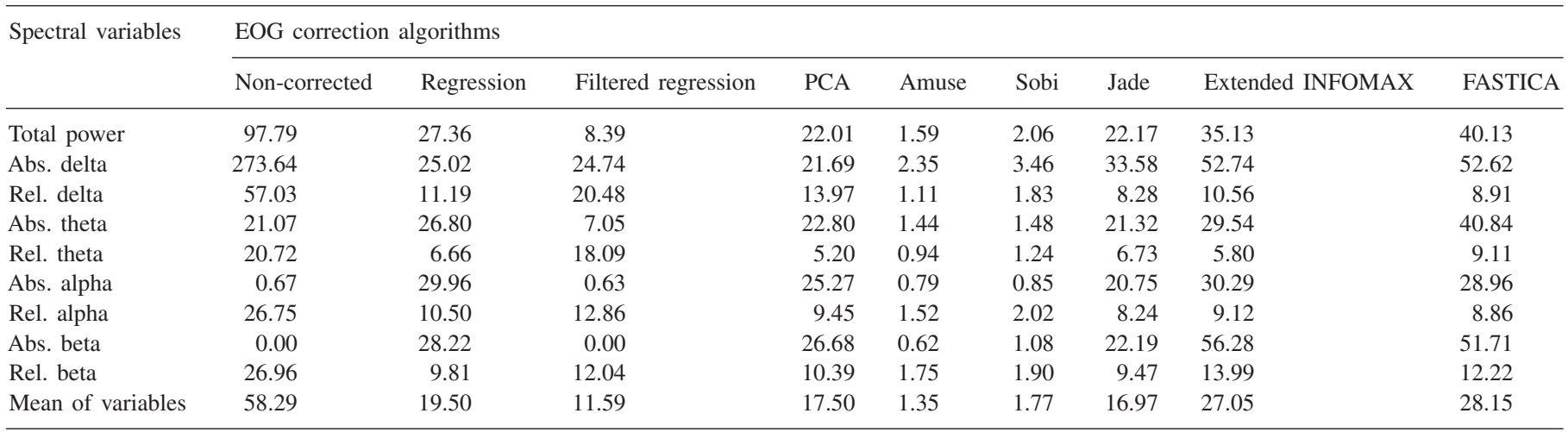

Mean of all EEG channels and mean of all the simulated subjects. 


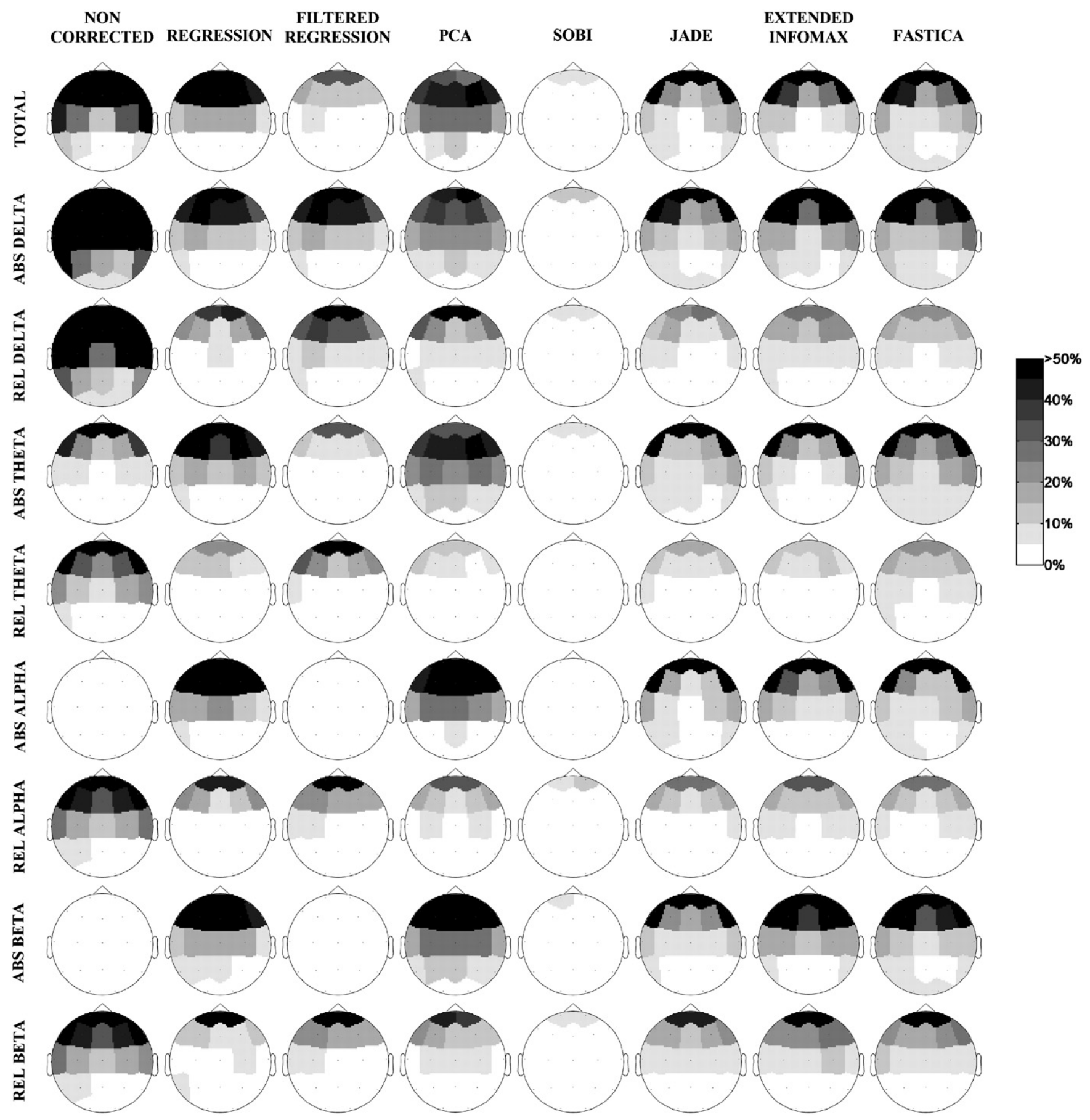

Fig. 6. Topographic maps of the errors (\%) between the sources and the corrected EEG signals by means of different ocular correction procedures. Errors (\%) between sources and mixed (non-corrected) signals are included in the first column. Color key is discretized from white to black in 5\% stripes. Errors higher than $50 \%$ are depicted as a dark red colour.

Errors with HOS techniques were generally higher than with the filtered regression-based approach in all variables, especially with INFOMAX and FASTICA algorithms. Regression, PCA and JADE provided similar errors. Regarding the error as an average of all variables, the lowest errors by far were obtained with AMUSE and SOBI. The former algorithm was the best approach because it provided a mean error below $2.5 \%$ for all spectral variables.

\subsubsection{Topographic maps}

The extent of ocular removal in each EEG channel was evaluated by representing the distribution of the spectral errors over the scalp. Fig. 6 shows the topographic maps of these percentage errors for each EOG correction procedure using the epoch duration which provided the minimum error (see Section 3.1). Observing the results for non-corrected EEG signals, the effect of ocular artifacts only was confirmed in delta and 


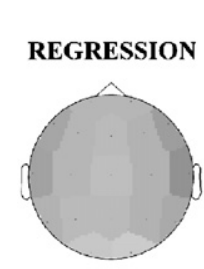

FILTERED

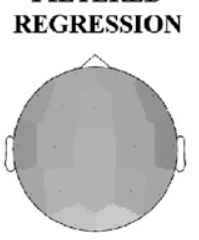

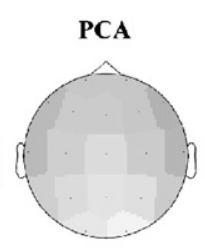
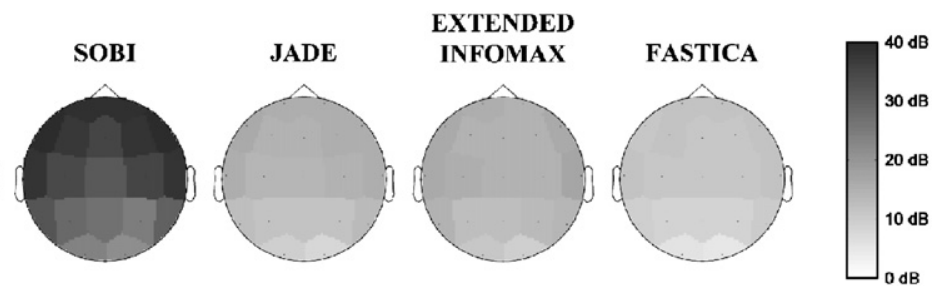

Fig. 7. Topographic maps showing improvements of SAR after applying the ocular artifact correction procedure. Best performances of the methods were selected.

Table 3

Percentage errors $(\%)$ in spectral variables for bss-based ocular reduction procedures

\begin{tabular}{|c|c|c|c|c|c|c|c|}
\hline Spectral variables & \multicolumn{7}{|c|}{ EOG correction algorithms } \\
\hline Total power & 97.79 & 23.93 & 12.89 & 10.72 & 35.3 & 32.51 & 48.61 \\
\hline Rel. delta & 57.03 & 22.9 & 12.68 & 13.25 & 14.89 & 11.23 & 17.08 \\
\hline Abs. theta & 21.07 & 23.82 & 7.12 & 6.38 & 34.03 & 27.41 & 43.62 \\
\hline Rel. theta & 20.72 & 10.21 & 6.66 & 6.29 & 9.87 & 7.34 & 11.76 \\
\hline Abs. beta & 0.00 & 26.67 & 6.76 & 6.49 & 37.75 & 43.19 & 48.33 \\
\hline Rel. beta & 26.96 & 14.68 & 7.10 & 6.82 & 12.97 & 10.57 & 12.01 \\
\hline Mean of variables & 58.29 & 22.03 & 10.89 & 9.95 & 26.83 & 23.98 & 33.02 \\
\hline
\end{tabular}

No EOG information-mean of all EEG channels and mean of all the simulated subjects.

theta bands. Topographic results were coherent with the propagation of eye movements over the scalp. Maximum errors were located at anterior sites, especially in fronto-polar and lateral-frontal channels. Although errors for absolute powers were similar between regression and ICA techniques at frontopolar and lateral-frontal channels, lower values were obtained for the latter in relative powers. The high errors for absolute alpha power showed that regression and PCA techniques removed more cerebral activity than the other methods. This was related to the bidirectional mixing simulation approach which introduced interferences in the broadband spectral power of the mixed EOG signals.

Comparing regression results with their filtered version, an improvement with the latter was observed in absolute variables related to theta and alpha bands. AMUSE and SOBI algorithms presented lower percentage errors than other correction approaches in all spectral variables. Errors in the fronto-polar channels were low, around 5\%, and they were even lower in the remaining channels.

Another index to quantify the performance of the ocular correction techniques was the improvement of SAR. Initial SAR for each channel before filtering was obtained using Eq. (6). Final SAR after filtering was calculated by the quotient between energy of true EEG source and MSE corresponding to corrected EEG signal and EEG source

Final $\operatorname{SAR}_{\mathrm{EEG}}($ in $\mathrm{dB})=10 \log \frac{\operatorname{Energy}\left\{\mathrm{EEG}_{\mathrm{s}}\right\}}{\left.\mathrm{MSE}_{2 \mathrm{EEG}}-\mathrm{EEG}_{\mathrm{s}}\right\}}$
The improvement of SAR $(\triangle \mathrm{SAR})$ was obtained subtracting the final SAR from the initial SAR.

$\Delta \operatorname{SAR}_{\mathrm{EEG}}($ in $\mathrm{dB})=10 \log \frac{\mathrm{MSE}\left\{\mathrm{EEG}_{\mathrm{m}}-\mathrm{EEG}_{\mathrm{s}}\right\}}{\left.\mathrm{MSE}_{2 \mathrm{EEG}}-\mathrm{EEG}_{\mathrm{s}}\right\}}$.

Corrected EEG signals would be equal to the EEG source with an ideal filtering, so MSE between them would be zero and $\triangle$ SAR would tend to infinite. Fig. 7 shows the topographic maps corresponding to improvements of SAR for each channel obtained by means of the same ocular correction methods shown in Fig. 6. $\triangle \mathrm{SAR}$ reached up to $25 \mathrm{~dB}$ for lateral-frontal channels with both regressions based approaches, whereas improvement with PCA and HOS-based techniques were around $15 \mathrm{~dB}$ for practically all EEG channels. AMUSE and SOBI algorithms provided the highest improvements of SAR (range: from $21.4 \mathrm{~dB}(\mathrm{O} 2)$ to $41.7 \mathrm{~dB}(\mathrm{~F} 7))$.

\subsection{Other channel configurations and absence of EOG recordings}

One of the main advantages of applying BSS procedures with respect to linear regression methods is that EOG recordings are not necessary as reference channels. However, all the studies until now reported have incorporated these ocular signals in order to improve the decomposition and the automatic identification of the IC related to the ocular activity. The same quantitative evaluation procedure described in this work was proposed in order to study the performance of the ocular reduction techniques when EOG channels were not available. 


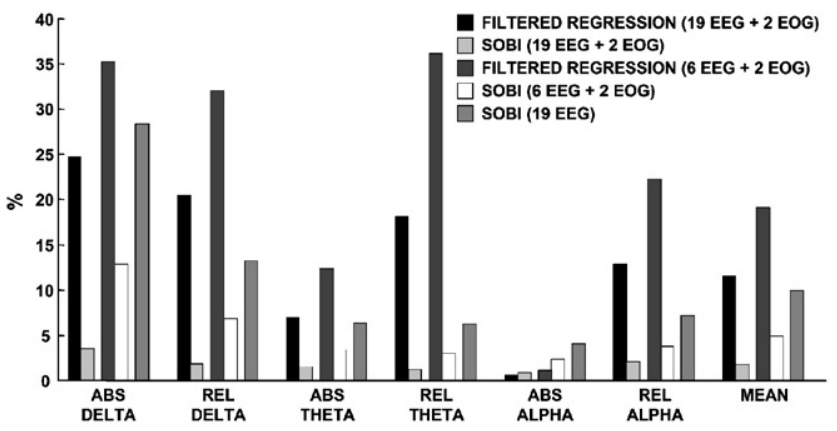

Fig. 8. A comparison of the percentage errors obtained for different EOG and EEG channel configurations in some spectral variables. Mean values were calculated from averaging all the nine target variables.

Source components were estimated by using only the 19 EEG channels in the BSS procedure. In this case, the algorithm proposed in this paper for the automatic ocular artifact identification was modified by replacing the EOG electrodes with the frontopolar (if vertical movement) or lateral-frontal (if horizontal) channels in the second criterion. Table 3 shows average percentage errors obtained for different ocular reduction methods using the same epoch duration as in Table 2. Comparing results from both tables, errors for every BSS-based method not using EOG recordings were logically higher than those obtained when EOG channels were included in the source decomposition. However, error values for SOBI algorithm without considering EOG channels were much lower than those obtained with the gold standard method (regression approach) and even slightly lower in average $(9.95 \%)$ than those with the filtered regression procedure $(11.59 \%)$.

On the other hand, a reduced or limited number of EEG electrodes is often used in sleep studies [28] and when subjects are neonates or young infants, due to the size of the head [29]. For the analysis of these situations and the comparison of these methods with a small number of EEG channels, a configuration including only six EEG electrodes (Fp1, Fp2, C3, C4, O1 and O2) was considered. In this case, filtered regression method and SOBI algorithm were applied to mixed multichannel data (two EOG and six EEG). Fig. 8 shows a comparison of the percentage errors in several target variables obtained for the different EEG and EOG channel configurations studied after applying both approaches and using an epoch duration of $90 \mathrm{~s}$ (similar values were obtained for AMUSE and SOBI). Results showed that percentage errors became higher in each method using a configuration with few EEG electrodes, but they kept in average much lower for SOBI algorithm than for filtered regression.

\section{Discussion and conclusion}

Ocular contamination of EEG data is an important and a very common problem in the diagnosis of neurobiological events because of the overlap between EEG and EOG in frequencies which can lead to wrong conclusions. Several techniques have been proposed to remove eye movement artifacts from spontaneous EEG signals based on regression analysis and BSS. For component-based methods, a new automatic procedure using logical rules, which are based on spectral and topographical information, is presented in order to identify the components related to eye activity. Results showed that all methods were able to reduce eye activity which contaminated EEG data. Evaluation of the performance of these correction techniques is difficult because of the inability to directly measure the electric activity of eyes and brain separately. To quantify the correction success of a method it is necessary to work with simulated EEG signals reproducing standard clinical situations that are as real as possible. The comparative study is based on spectral variables and topographic maps that are commonly used in clinical routine.

Ocular removal procedures based on regression analysis are considered as the gold standard method in spontaneous EEG signals [16] because they provide a relatively simple mathematical solution by removing the ocular component from EEG channels. However, a partial neural component is also subtracted by the regression process due to the mutual contamination between EEG and EOG channels. In this study, this was shown by the corrected signals from the fronto-polar channels (see Fig. 4) and by the high errors obtained for absolute alpha power $(29.96 \%)$. This neural removal was partly reduced by applying a low-pass filter to the EOG channels before the subtraction. Errors with filtered regression were almost zeros for absolute power in fast bands (alpha and beta) since no ocular contamination was included by the simulation procedure. However, worse results were shown for relative powers of these bands (around 12\%, see Table 2).

Smaller errors were obtained for PCA using short duration data (see Fig. 5), although they were not lower than for regression (see Fig. 6). PCA does not use a realistic physical solution since there is no reason to assume that EOG and EEG sources are orthogonal. To overcome this unrealistic constraint, other SOS and HOS techniques have appeared which decomposed the sources more properly.

HOS-based techniques use statistic information as higher order cumulants, entropy or maximum likelihood estimation in order to separate the sources by minimizing an approximation of mutual information. They are composed of time consuming iterations for optimizing certain HOS-based cost functions. HOS-based algorithms required a certain data length. Delorme et al. [13] recommend at least a number of samples corresponding to a few times $(k)$ the square of the number of channels. In our case, $k \cdot 21^{2}=k \cdot 441$ samples were needed, where $k$ is an integer. Results showed that errors decreased with higher data length and tended to similar values to those obtained with PCA when increasing data length. Although the lowest errors were obtained with an epoch duration of $180 \mathrm{~s}$ (18000 samples which corresponded to $k=40$ ), similar values to these minima were already obtained with $90 \mathrm{~s}$ (9000 samples which corresponded to $k=20$ ). Stationarity of the spatial structure of the sources has to be assumed when a long data epoch is used, because these approaches would not be appropriated in another case. 
Separation by SOS and HOS-based algorithms was not expected to be similar because they have different assumptions about sources modeling. Sources extracted by HOS-based methods are generally noisier than sources extracted by SOS techniques. AMUSE and SOBI algorithms use temporal structure of mixed EEG signals to achieve the blind separation. These spatio-temporal decorrelation procedures are simple, fast to compute and robust. Besides, they were not sensitive to the duration of data in the present study. Similar errors were obtained for all epoch durations and for both approaches (see Fig. 5). Time and frequency results indicated that these SOS-based ocular removal techniques preserved and recovered more brain activity than the other methods. Best ocular corrected EEG signals were obtained using them for blind separation: errors lower than 5\% for all spectral variables at anterior sites (lower than $2.5 \%$ as an average of all channels) and the highest SAR improvements up to $40 \mathrm{~dB}$ at these anterior sites.

In clinical routine, electrode configurations change depending on the application. Moreover, in last years, many algorithms using different number of EEG and EOG electrodes have been suggested in order to reduce ocular artifacts from EEG data and opposite conclusions were obtained. Up to date, some studies proposed BSS algorithms [9,10], others defended regression analysis, especially when the number of EEG channels is small $[12,14]$. Although the main part of this study was performed using 19 EEG and 2 EOG channels according to the international 10-20 system, a comparison between methods was also carried out with only six EEG electrodes and even in the worst situation: without EOG recordings. EOG signals are absolutely necessary for regression-based approaches, but not for BSS procedures. However, the studies in the literature focused the automatic ocular identification on the correlation between an IC source and the EOG channels. Considering only six EEG + two EOG channels, errors in spectral parameters obtained for SOBI technique were much lower than those calculated by regression-based methods. Even, errors for SOBI algorithm considering only 19 EEG channels (without EOG) were slightly lower than those obtained with filtered regression approach but recording additionally two EOG channels in the latter.

Therefore, based on the effects quantified on clinical target variables and applying fully automated BSS procedure in simulated EEG and EOG recordings, we conclude that AMUSE and SOBI are the most suitable algorithms to be used in clinical and functional EEG studies, not only independently of the number of EEG leads, but also when EOG recordings are not available.

\section{Conflict of interest statement}

None declared.

\section{Acknowledgment}

This study was partially supported by CICYT (TEC200402274) from Spain.

\section{References}

[1] O.G. Lins, T.W. Picton, P. Berg, M. Scherg, Ocular artifacts in EEG and event-related potentials I: scalp topography, Brain Topogr. 6 (1993) $51-63$.

[2] T.-P. Jung, S. Makeig, M. Westerfield, J. Townsend, E. Courchesne, T.J. Sejnowski, Removal of eye activity artifacts from visual event-related potentials in normal and clinical subjects, Clin. Neurophysiol. 111 (2000) $1745-1758$.

[3] T. Gasser, P. Ziegler, F. Gattaz, The deleterious effect of ocular artifacts on the quantitative EEG, and a remedy, Eur. Arch. Psychiatry Clin. Neurosci. 241 (1992) 241-252.

[4] D. Hagemman, E. Naumann, The effects of ocular artifacts on (lateralized) broadband power in the EEG, Clin. Neurophysiol. 112 (2001) 215-231.

[5] O.G. Lins, T.W. Picton, P. Berg, M. Scherg, Ocular artifacts in recording EEGs and event-related potentials II: Source dipoles and source components, Brain Topogr. 6 (1993) 65-78.

[6] T.D. Lagerlund, F.W. Sharbrough, N.E. Busacker, Spatial filtering of multichannel electroencephalographic recordings through principal component analysis by singular value decomposition, J. Clin. Neurophysiol. 14 (1997) 73-82.

[7] S. Makeig, A.J. Bell, T.-P. Jung, T.J. Sejnowski, Independent component analysis of electroencephalographic data, Adv. Neural Inf. Process. Syst. 8 (1996) 145-151.

[8] R.N. Vigario, Extraction of ocular artifacts from EEG using independent component analysis, Electroencephalogr. Clin. Neurophysiol. 103 (1997) $395-404$.

[9] C.A. Joyce, I.F. Gorodnitsky, M. Kutas, Automatic removal of eye movement and blink artifacts from EEG data using blind component separation, Psychophysiology 41 (2004) 313-325.

[10] J.J.M. Kierkels, G.J.M. van Boxtel, L.L.M. Vogten, A model-based objective evaluation of eye movement correction in EEG recordings, IEEE Trans. Bio-Med. Eng. 53 (2006) 246-253.

[11] G. Barbati, C. Porcaro, F. Zappasodi, P.M. Rossini, F. Tecchio, Optimization of an independent component analysis approach for artifact identification and removal in magnetoencephalographic signals, Clin. Neurophysiol. 115 (2004) 1220-1232.

[12] G.L. Wallstrom, R.E. Kass, A. Miller, J.F. Cohn, A.F. Nathan, Automatic correction of ocular artifacts in the EEG: a comparison of regressionbased and component-based methods, Int. J. Psychophysiol. 53 (2004) $105-119$.

[13] A. Delorme, S. Makeig, EEGLAB: an open source toolbox for analysis of single-trial EEG dynamics including independent component analysis, J. Neurosci. Methods. 134 (2004) 9-21.

[14] A. Schlögl, C. Keinrath, D. Zimmermann, et al., A fully automated correction method of EOG artifacts in EEG recordings, Clin. Neurophysiol. 118 (2007) 98-104.

[15] H. Jasper, The ten-twenty electrode system of the International Federation, Electroencephalogr. Clin. Neurophysiol. 10 (1958) 371-375.

[16] P. Anderer, H.V. Semlitsch, B. Saletu, M.J. Barbanoj, Artifact processing in topographic of electroencephalographic activity in neuropsychopharmacology, Psychiatry. Res. 45 (1992) 79-93.

[17] H.V. Semlitsch, P. Anderer, P. Schuster, O. Presslich, A solution for reliable and valid reduction of ocular artifacts applied to the P300 ERP, Psychophysiology 23 (1986) 695-703.

[18] L. Tong, R.-W. Liu, W.C. Soon, Y.-F. Huang, Indeterminacy and identifiability of blind identification, IEEE Trans. Circuits Syst. 5 (1991) 499-509.

[19] A. Belouchrani, K. Abed-Meraim, J.F. Cardoso, E. Moulines, A blind source separation technique using second-order statistics, IEEE Trans. Signal Process. 45 (1997) 434-444.

[20] A. Hyvärinen, J. Karhunen, E. Oja, Independent Component Analysis, Wiley, New York, 2001.

[21] J.F. Cardoso, A. Souloumiac, Blind beamforming for non Gaussian signals, IEE Proc.-F. 140 (1993) 362-370.

[22] A.J. Bell, T.J. Sejnowski, An information maximization approach to blind separation and blind deconvolution, Neural Comput. 7 (1995) 1129-1159. 
[23] A. Hyvärinen, E. Oja, A fast fixed-point algorithm for independent component analysis, Neural Comput. 9 (1997) 1483-1492.

[24] T.-W. Lee, M. Girolami, T.J. Sejnowski, Independent component analysis using an extended infomax algorithm for mixed sub-Gaussian and superGaussian sources, Neural Comput. 11 (1999) 606-633.

[25] A. Cichocki, S. Amari, K. Siwek, T. Tanaka et al., ICALAB toolboxes [online], Available from 〈http://www.bsp.brain.riken.jp/ICALAB/〉 (accessed 15 Feb 2007).

[26] R.J. Croft, R.J. Barry, Removal of ocular artifact from the EEG: a review, Neurophysiol. Clin. 30 (2000) 5-19.

[27] B. Saletu, P. Anderer, K. Kinsperger, J. Grünberger, Topographic brain mapping of EEG in neuropsychopharmacology-Part II. Clinical applications (pharmaco EEG mapping), Methods Findings Exp. Clin. Pharmacol. 9 (1987) 385-408.

[28] E. Huuponen, S.-L. Himanen, A. Värri, J. Hasan, et al., A study on gender and age differences in sleep spindles, Neuropsychobiology 45 (2002) 99-105.

[29] P. Celka, B. Boashash, P. Colditz, Preprocessing and time-frequency analysis of newborn EEG seizures, IEEE Eng. Med. Biol. Mag. 20 (2001) 30-39.

Sergio Romero received his Industrial Engineering M.Sc. degree from the Technical University of Catalonia (UPC) in 2000. Subsequently, he is pursuing his Ph.D. degree in Biomedical Engineering at UPC. He is currently an
Assistant Professor of the Department of Automatic Control and Systems Engineering (ESAII) at the same university. His current research interest is biomedical signal processing focused in spectral estimation, time-frequency representation, blind source separation and adaptive algorithms.

Miguel Angel Mañanas received his Telecommunications Engineering and Ph.D. in Biomedical Engineering degrees from the Technical University of Catalonia (UPC) in 1993 and 1999, respectively. He is currently an Associate Professor and Vice-director on Research at the Department of Automatic Control and Systems Engineering (ESAII) at the same university. $\mathrm{He}$ is a member of the Biomedical Engineering Research Center (CREB, UPC) and the Spanish Committee from the International Federation of Automatic Control (CEA). His active research areas include biomedical signal processing, statistical analysis, modeling and simulation. His expertise is specifically in spectral estimation, adaptive algorithms, time-frequency representations, respiratory control system, independent component analysis, and nonlinear techniques applied to EMG, MMG, EEG and respiratory signals.

Manuel José Barbanoj is the head of the Drug Research Center (CIM) of the Research Institute of Sant Pau Hospital (Barcelona). He is currently an Associate Professor at the Department of Pharmacology and Therapeutics at the Autonomous University of Barcelona (UAB). He has wide experience in carrying out Phase I clinical studies in the psychopharmacological field concerning the implementation of neurophysiological measures such as quantitative pharmaco-EEG, evoked potentials and polygraphic sleep recordings. Special focus is paid to PK-PD modeling (combination of drugs, pharmacokinetics and pharmacodynamics in order to enlarge pathophysiological knowledge). 